\title{
Spatial mapping of temporal risk characteristics to improve environmental health risk identification: A case study of a dengue epidemic in Taiwan
}

\author{
Tzai-Hung Wen ${ }^{\mathrm{a}}$, Neal H. Lin ${ }^{\mathrm{b}}$, Chun-Hung Lin ${ }^{\mathrm{a}}$, \\ Chwan-Chuen King ${ }^{\mathrm{b}}$, Ming-Daw $\mathrm{Su}^{\mathrm{a}, *}$ \\ ${ }^{a}$ Department of Bioenvironmental Systems Engineering, National Taiwan University, Taipei, Taiwan \\ ${ }^{\mathrm{b}}$ Institute of Epidemiology, College of Public Health, National Taiwan University, Taipei, Taiwan \\ Received 24 November 2005; received in revised form 6 February 2006; accepted 7 February 2006 \\ Available online 11 April 2006
}

\begin{abstract}
Mapping uneven events, such as disease cases or pollutants, is a basic but important procedure for analyzing regional relationships and variation in public health and environmental agencies. The purpose of mapping is to find out the spatial clustering of uneven events and identify spatial risk areas, which could lead to potential environmental hazards or epidemics. Meanwhile, more hypotheses could be generated through mapping process for further investigations. This paper proposed a novel spatial-temporal approach to focusing on: (1) how often these uneven cases occur, (2) how long these cases persist and (3) how significant cases occur in consecutive periods across the study area. The proposed model was applied to the dengue fever epidemic in Taiwan in 2002 as a case study, which was the worst epidemic in the last 60 years. This approach provides procedures to identify spatial health risk levels with temporal characteristics and assists in generating hypothesis that will be investigated in further detail.
\end{abstract}

(C) 2006 Elsevier B.V. All rights reserved.

Keywords: Spatial risk assessment; Spatial-temporal clustering; Geographic information systems (GIS)

\section{Introduction}

Mapping uneven events, such as disease cases or pollutants, makes it possible for public administrators to discover the origin of pollution or source of

\footnotetext{
* Corresponding author. Department of Bioenvironmental Systems Engineering, National Taiwan University, No. 1, Sec. 4, Rd. Roosevelt, Taipei City, Taiwan 10617. Tel.: +886 22363 1108; fax: +886223635854 .

E-mail address: sumd@ntu.edu.tw (M.-D. Su).
}

epidemic outbreaks and generate more hypotheses for further investigations. More importantly, it may spatially identify the high-risk areas, which can be targeted for environmental hazards and public health prevention. Mapping has been made easier and become more wisely used with the development of geographical information systems (GIS). However, mapping provides only a visual display of the uneven cases, but cannot definitively confirm clustering of cases or spatial correlations. Spatial statistics is extensively used to find relationship of case rates 
and their geographical location (Croner et al., 2000). Point pattern analysis is a method often employed in the detection of clustering patterns. Since points may represent actual locations of uneven events, the method involves in the issue of privacy (Maheswan and Haining, 2004). Risk surface estimation, including kernel estimation and geostatistical methods, produces continuous surfaces of risk across the whole study areas and potentially offer more insight into the nature of the clusters (Bithell, 1990, 1999; Diggle, 2000). However, mapping the spatial clusters of uneven events is a static snapshot, which ignores the temporal kinetics of these uneven events, and it is difficult to evaluate whether the hazards or epidemics have been broken out or kept under control by policymakers. Time-series statistical techniques, such as Box-Jenkins (seasonal) autoregressive integrated moving average (ARIMA) models, are used to forecast the outbreak of uneven cases or to estimate the expected incidence values (Zeger et al., 2004; Earnest et al., 2005). But these kinds of approaches could not provide clues for identification of spatial risk areas. This paper intends to propose a spatialtemporal risk model for mapping geographic distribution of uneven events with temporally defined indices to improve health risks identifications. The model focuses on three temporal risk characteristics across geographic space: (1) how often these uneven cases occur, (2) how long these cases persist and (3) how significant cases occurring in consecutive periods. This model will be applied to the dengue fever epidemic in Taiwan in 2002 as a case study, which is the worst epidemic in the last 60 years.

Dengue is a member of flavivirus with four known antigenically distinct serotypes and is vectored mainly by domestically adapted mosquito species, specifically Aedes aegypti. Environmental risk factors, including water storage containers, housing patterns and frequency of waste disposal, etc., reflect the condition of environmental health in certain area (Bohra and Andrianasolo, 2001). These risk factors could have potential to cause more mosquito breeding sites, which might lead to an outbreak of dengue epidemic. However, routinely monitoring environmental risk factors is not feasible for public health and environment agencies. Therefore, this study only used dengue caseincidence data, including onset date of a confirmed dengue case and its geographic location, collected by official surveillance systems to investigate whether the approach will improve the detection of environmental health risk areas and the evaluation of vector or mosquito control measures.

\section{Materials and methods}

\subsection{Study area and data}

Kaohsiung City, located in southern Taiwan, is the second largest metropolitan city, the largest commercial harbor and an important land, marine and air transportation hub. Fig. 1 shows the location of Kaohsiung City and one of its satellite cities, Fengshan City. This twocity area was used as our study area because it was the major dengue epidemic foci in southern Taiwan. The study focused on dengue cases based on surveillance data, which reported 4790 confirmed dengue cases (DEN), including 4574 dengue fever (DF) cases and 216 dengue hemorrhagic fever (DHF) cases, from April to December in the year of 2002 (Wu et al., 2003). The registered population for this two-city area was used as the control population. Fig. 2 illustrates the temporal progression of DEN and DHF cases for the 2002 calendar-year, but it gave no clues on the interplay between temporal and the spatial distribution of these cases.

\subsection{Spatial and temporal units to summarize case- incidence data}

A Li, the smallest administrative unit in Taiwan, was used as the spatial mapping unit. This study involved $542 \mathrm{Li}$, and most $\mathrm{Li}$ in urban region cover an area of between 0.26 and $0.58 \mathrm{~km}^{2}$, and have $2100-5300 \mathrm{popu}-$ lations or 850-1600 households (DGBAS, 2005).

The Center for Disease Control-Taiwan (CDCTaiwan) summarized dengue cases on a weekly basis. Therefore, our study used a week as temporal unit for better comparison on different indices. Case data were provided by the CDC-Taiwan. Personal identifiers were removed for privacy concern but the spatial locations were preserved for mapping and analysis purposes. These data were then summarized by $\mathrm{Li}$ to avoid the exact locations of patients and thus further protect their privacy.

\subsection{Temporal risk indices}

The occurrence of disease can be described as either a sporadic or clustering event, both temporally and spatially. An infectious disease epidemic may result from intensive contact with the contagion, the environment or other risk factors depending on the mode of transmission for that specific disease (Giesecke, 2001). A number of indicators are used to measure the magnitude and severity of an epidemic, 


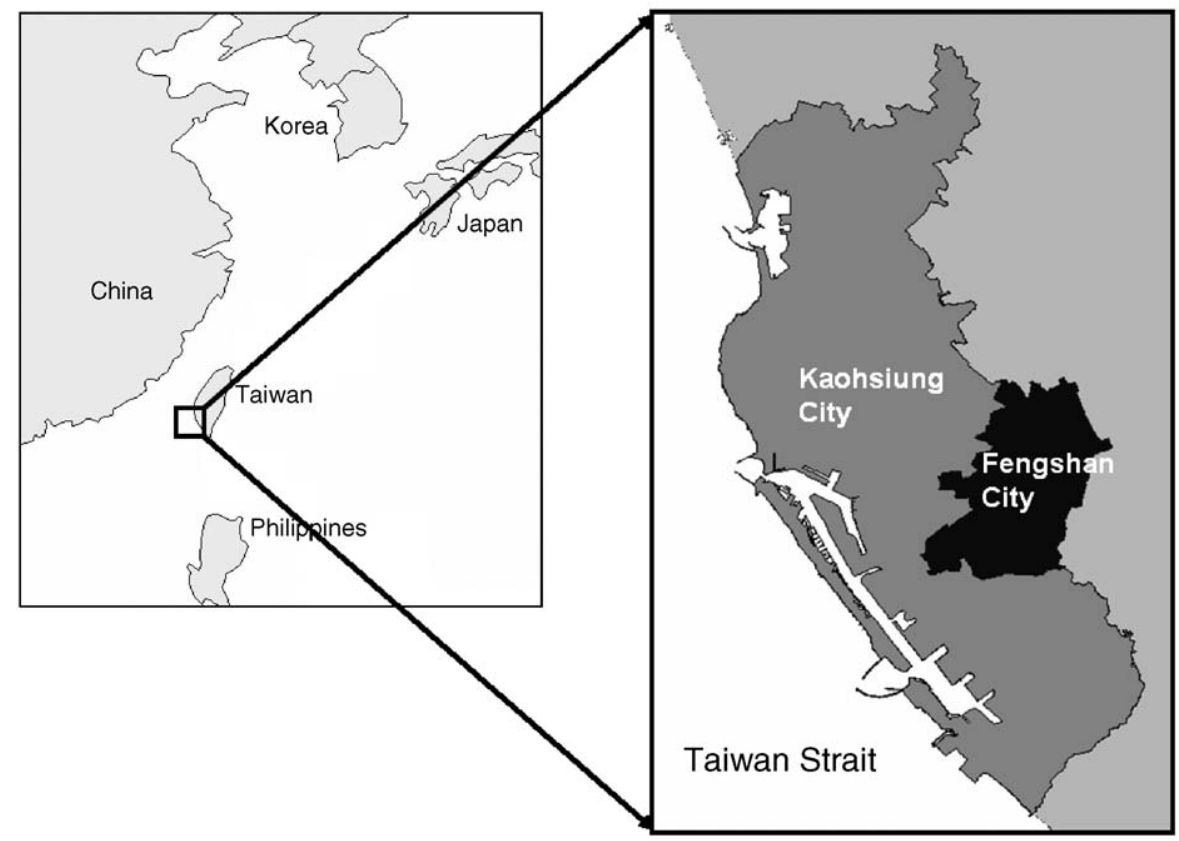

Fig. 1. Geographic distribution of Kaohsiung and Fengshan cities (two-city area) where the major dengue epidemic foci in southern Taiwan, 2002.

with annual incidence rate being the most commonly used index in the measurement of risk (Dunn et al., 2001). Fig. 3 shows two hypothetical epidemic patterns for the same area that have the same annual incidence rate. The cases in Fig. 3(A) were concentrated between week 29 and week 45, while those in Fig. 3(B) occurred sporadically throughout the year. From visual inspection of the two epidemic curves, the two different patterns implied that two different control strategies were needed. But annual incidence rate, as a risk index, cannot differentiate the epidemic severities of these two temporal patterns. Thus, temporal factors are important and should be taken into account in dealing with environmental risk identification. This paper will define three temporal indices to evaluate the severity and magnitude of an epidemic risk for (1) how often the disease occurs (frequency), (2) how long an epidemic persists (duration) and (3) how significant cases occurring in consecutive weeks are during an epidemic (intensity).

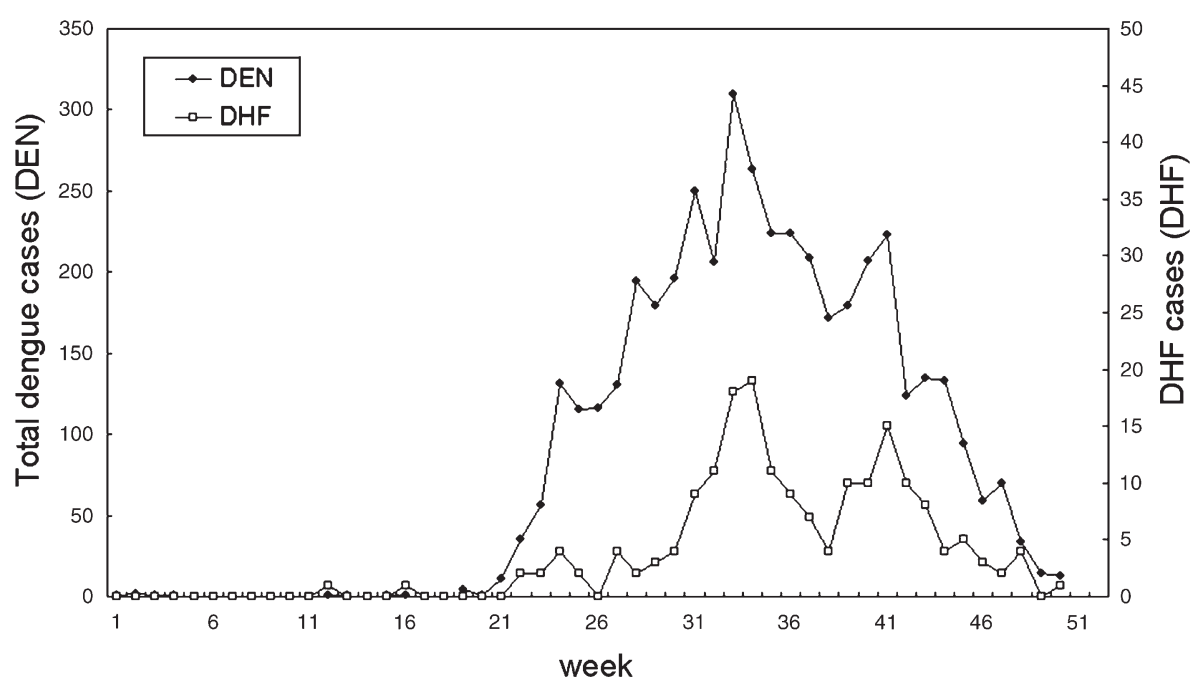

Fig. 2. Epidemic curve of dengue in Kaohiung and Fon-Shan cities, 2002. 

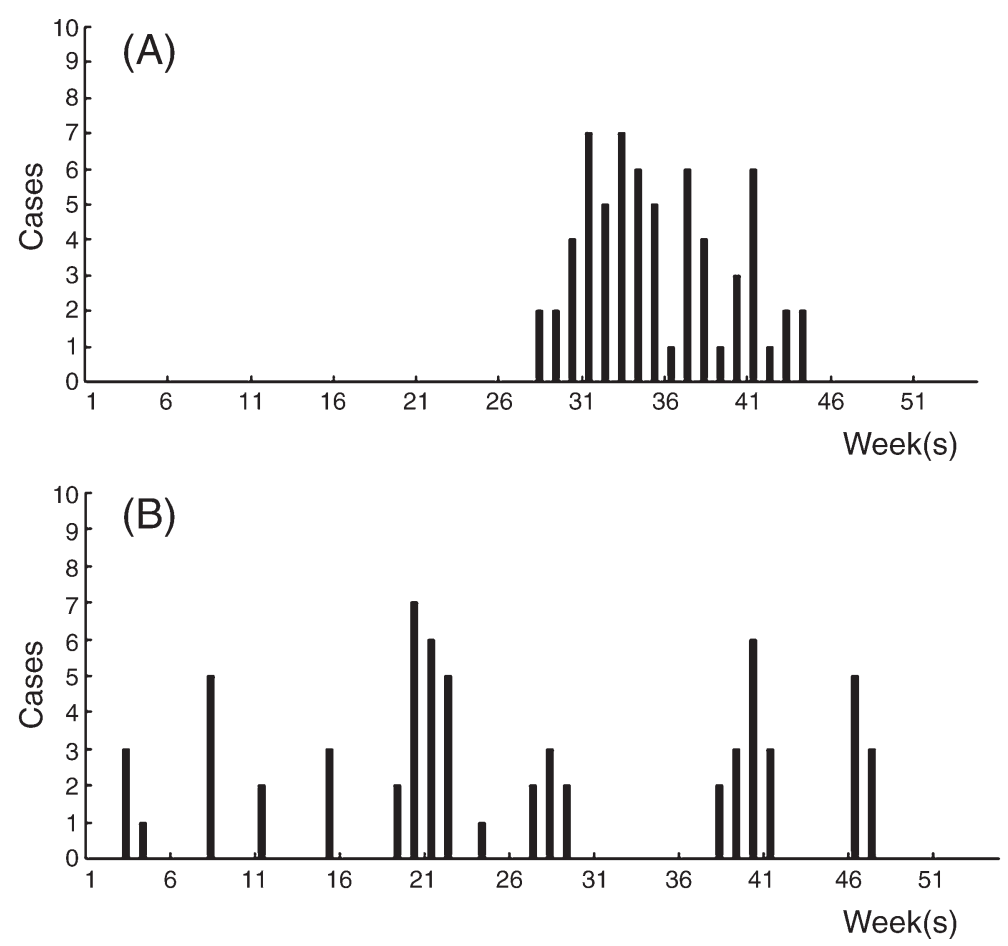

Fig. 3. Two hypothetical epidemic patterns with different temporal risk characteristics but the identical annual incidence rate.

\subsection{Frequency index $(\alpha)$}

The frequency of a disease during an epidemic can be described by a probability that one or more laboratory confirmed cases occurred in certain week(s) out of the total epidemic period. The frequency index $(\alpha)$ can be defined as:

$\alpha=\frac{\mathrm{EW}}{\mathrm{TW}}$

where TW is the total number of weeks during the entire epidemic period. EW is the total number of weeks which one or more cases occurred during the entire epidemic period. The value of the frequency index $(\alpha)$ ranges between 0 and 1 . As the value of the frequency index approaches to 1 , the possibility of disease occurrence in certain week(s) increases.

\subsection{Duration index $(\beta)$}

With the knowledge of a disease occurrence frequency, it is still hard to recognize whether the case(s) appear suddenly and then disappear rapidly or persist for a certain period of time in an interested geographical area. The duration of an epidemic usually consists of several epidemic waves. An epidemic wave is defined in this paper as the number of weeks when cases successively occur. These weeks, even with only one case occurs, are also important and are considered as part of the epidemic wave. The average number of weeks of an epidemic wave can be used as the duration index for the entire epidemic period. Thus, the duration index of an epidemic can be described as the average number of weeks in which occurrence of cases persists during the whole epidemic period. This index $(\beta)$ is defined as

$\beta=\frac{\mathrm{EW}}{\mathrm{EV}}$

where $\mathrm{EW}$ is described above and $\mathrm{EV}$ is the total number of epidemic waves during the entire epidemic period. The duration index $(\beta)$ is very important for practitioners and administrators of public health and environment agencies because it reflects the effectiveness of the prevention or control strategies used during the epidemic. A larger value of $\beta$ indicates that the cases are less likely to disappear once they occur and more chance to result in virus mutation.

\subsection{Intensity index $(\gamma)$}

Intensity refers to the likely magnitude within an epidemic wave when more than one case occurs. 
Incidence rate is taken as an index to measure the magnitude of new cases appearing during a specified period. However, it cannot measure the weekly severity during the period. Therefore, the intensity index $(\gamma)$ is formulated as:

$\gamma=\frac{\mathrm{IR}}{\mathrm{EV}}$

where IR is the incidence rate during the defined epidemic period and EV is as described above. The index evaluates the severity of an epidemic by focusing on successive weeks when cases have occurred. A high $\gamma$ value describes an epidemic that is more temporally concentrated. The value of $\gamma$ will become smaller if there were more waves, as most cases are temporally dispersed throughout the epidemic.

The two hypothetical epidemic patterns in Table 1 can be used to show how the three temporal indices differ in despite of having the identical annual incidence rate. For the dengue epidemic described by these two situations, situation (a) would be more temporally significant. The frequency index $\alpha$ of situation (b) is slightly higher than that of situation (a) $[35.2 \%$ vs. $31.5 \%$, meaning that there are a greater number of weeks with at least one case occurring. However, the duration index $\beta$ and intensity index $\gamma$ of situation (a) is higher than those of situation (b) $[\beta: 17$ vs. $2.1, \gamma$ : 0.0064 vs. 0.00071$]$, meaning that the epidemic of situation (b) has shorter epidemic waves and milder intensity. Overall, the situation (a) had higher frequency index than situation (b) but shorter duration and less intensity even though both situations have the same incidence. Therefore, we may conclude that the epidemic patterns of situation (a) is more severe than situation (b) from these three temporal indices and provide more public health resources and exercise more control measures for situation (a).

\subsection{Identification of significant risk areas}

Epidemiologists used to calculate odds ratios (ORs) with the specific confidence interval (CI) as relative risk measures to assess the significant risk factors (Duarte et al., 2006; Wilder-Smith et al., 2004). Rather than these case-control studies, local spatial autocorrelation index is used in this study to evaluate significant clustering trends of an interested temporal index as spatial risk areas. The local indicator of spatial autocorrelation (LISA) was adopted as the spatial risk index to identify significant spatial clusters (Anselin, 1995). The definition of LISA index is given below:

$I(i)=\frac{\left(X_{i}-\bar{X}\right)}{\delta} \times \sum_{j=1}^{n}\left[W_{i j} \times \frac{\left(X_{j}-\bar{X}\right)}{\delta}\right]$

where $I(i)=$ the LISA index for region $i, W_{i j}=$ the proximity of region $i$ to region $j, X_{i}=$ the value for the temporal index of region $i, X_{j}=$ the value for the temporal index of region $j, \bar{X}=$ the average value of the temporal index, $\delta=$ the standard deviation of $X_{i}$ and $n=$ the total number of the regions to be evaluated.

The term $W_{i j}$ describes the proximity of region $i$ to $j$. If the region $i$ is next to region $j$, a value of 1 is assigned; 0 otherwise. For any region $i$ next to region $j$, the term $\left(X_{i}-\bar{X}\right) \times\left(X_{j}-\bar{X}\right)$ describes the degree of similarity in a tested index within a designated area and its neighbors. Monte Carlo significance test, which calculate the likelihood that clusters could have arisen by chance in a given population, was used to evaluate the statistical significance of spatial clusters. Thus, each of the temporal indices for the 2002 dengue epidemic in the two-city area was identified as high with a $95 \%$ statistical significance.

\section{Results}

\subsection{Spatial pattern analysis of temporal risk indices}

Since any epidemic is a dynamic process, it would be important to identify spatial risk areas, if any, through the use of different temporal characteristics. For example, some area might have longer epidemic duration and the others might have stronger intensity even though the duration is short. Once the risk areas at different epidemic periods are identified, the comparisons of case-incidence with its temporal-spatial dynamics can be helpful in identifying the possible causes or effective control measures.

Table 1

Temporal risk indices between case (a) and case (b) in the two hypothetical epidemic patterns

\begin{tabular}{lllllll}
\hline & At-risk population & Infected cases & Annual incidence rate & Frequency index, $\alpha$ & Duration index, $\beta$ & Intensity index, $\gamma$ \\
\hline Case (a) & 10,000 & 64 & 0.0064 & $31.5 \%$ & 17.0 weeks & 0.0064 \\
Case (b) & 10,000 & 64 & 0.0064 & $35.2 \%$ & 2.1 weeks & 0.00071 \\
\hline
\end{tabular}


Table 2

Statistics of temporal risk indices cross the study areas

\begin{tabular}{lccc}
\hline & Frequency index, $\alpha$ & $\begin{array}{l}\text { Duration index, } \\
\beta \text { [week(s) per wave] }\end{array}$ & $\begin{array}{l}\text { Intensity index, } \gamma \\
\text { [incidence rate per wave] }\end{array}$ \\
\hline Count of Li & 542 & 542 & 542 \\
Mean $(Z$-score) & $0.086(0)$ & $1.40(0)$ & $0.807(0)$ \\
Maximum $(Z$-score) & $0.404(3.93)$ & $17.00(11.91)$ & $8.754(6.99)$ \\
Minimum $(Z$-score) & $0.000(-1.06)$ & $0.00(-1.07)$ & $0.000(-0.71)$ \\
Range (range of $Z$-score) & $0.404(4.99)$ & $17.00(12.98)$ & $8.754(7.70)$ \\
Standard deviation (S.D.) & 0.081 & 1.31 & 1.137 \\
Count of Li with statistically & 80 & 38 & 32 \\
$\quad$ significant high values $(p<0.05)$ & & & \\
\hline
\end{tabular}

To identify potential risk areas and analyze different spatial patterns using appropriate temporal risk indices, we first calculated the values of the three above defined temporal indices for each $\mathrm{Li}$ and its descriptive statistics across the study areas. These statistics were transformed into $Z$-scores for comparison on an identical basis as shown in Table 2. It could be found that the range of duration index has largest variation among these three indices (-1.07-11.91). It may imply that the existence of blind spots in implementing control measures resulted in persistent occurrence of DF virus in those areas. The counts of $\mathrm{Li}$ with significant high values of temporal indices are shown in the last row of Table 2. Even though the ratios of Li identified as statistically significant are low (80/542 for $\alpha, 38 / 542$ for $\beta$ and $32 /$ 542 for $\gamma$ ), public health administrators should pay more attention to those areas, because these risk areas with different temporal risk characteristics might result from control failure or poor conditions of environmental health, and so on. Therefore, analyzing the risk types in those areas could be helpful to identify possible reasons why the epidemic was out of control.

We then calculated the correlation among these three indices as shown in Table 3. It could be found that the values of these indices are highly correlated. For example, the correlation coefficient between intensity and duration is 0.75 . It could indicate higher number of

Table 3

Correlation of temporal risk indices

\begin{tabular}{|c|c|c|c|}
\hline & $\begin{array}{l}\text { Frequency } \\
\text { index, } \alpha\end{array}$ & $\begin{array}{l}\text { Duration index, } \\
\beta \text { [week(s) } \\
\text { per wave] }\end{array}$ & $\begin{array}{l}\text { Intensity index, } \\
\gamma \text { [incidence rate } \\
\text { per wave] }\end{array}$ \\
\hline Frequency index, $\alpha$ & 1.00 & $0.72^{\mathrm{a}}$ & $0.51^{\mathrm{a}}$ \\
\hline $\begin{array}{l}\text { Duration index, } \\
\beta \text { [week(s) } \\
\text { per wave] }\end{array}$ & & 1.00 & $0.75^{\mathrm{a}}$ \\
\hline $\begin{array}{l}\text { Intensity index, } \\
\gamma \text { [incidence rate } \\
\text { per wave] }\end{array}$ & & & 1.00 \\
\hline
\end{tabular}

\footnotetext{
${ }^{a}$ Correlation is significant at the 0.01 level.
}

dengue cases was found during a single epidemic wave, and then the cases were most likely to occur consecutively in later period. Then, it will be valuable for public health personnel to identify spatial risk areas where the dengue cases with longer duration or higher transmission intensity. Thus, the following analysis is to show the spatial distribution of these three temporal indices. Through mapping of each temporal risk characteristics, we can identify where those spatial risk areas were and then compared their different risk patterns.

Fig. 4 shows spatial distribution of the three temporal risk indices normalized by standard deviations (S.D.). It could be found that there are some risk areas with longer epidemic duration but lower transmission intensity, such as those in circle (a) in the panels (B) and (C). Those areas are easily ignored by health or environmental agencies due to lower transmission intensity. Contrarily, some areas has high transmission intensity but low epidemic duration, which implies control measures in those areas are effective to break the dengue transmission routes, such as those in circle (b) in the panels (B) and (C). Furthermore, some areas with high dengue cases frequency did not have high intensity, such as those in circle (c) in the panels (A) and (C), where many administrative $\mathrm{Li}$ cannot thoroughly eliminate the continuous occurrence of cases in this dengue epidemic. Contrarily, some risk areas have low frequency but high transmission intensity, implying that control measures in those areas could eliminate the vector breeding sites or dengue virus in the short-term periods, such as those in circle (d) in the panels (A) and (C). Thus, considering the combinations of temporal indices is important to differentiate risk characteristics of each $\mathrm{Li}$.

\subsection{Classification of spatial risk areas and interpreta- tions of the epidemic processes}

The risk maps for these three temporal indices were overlaid together for analysis of combination effects. Each Li was classified into one of the eight 

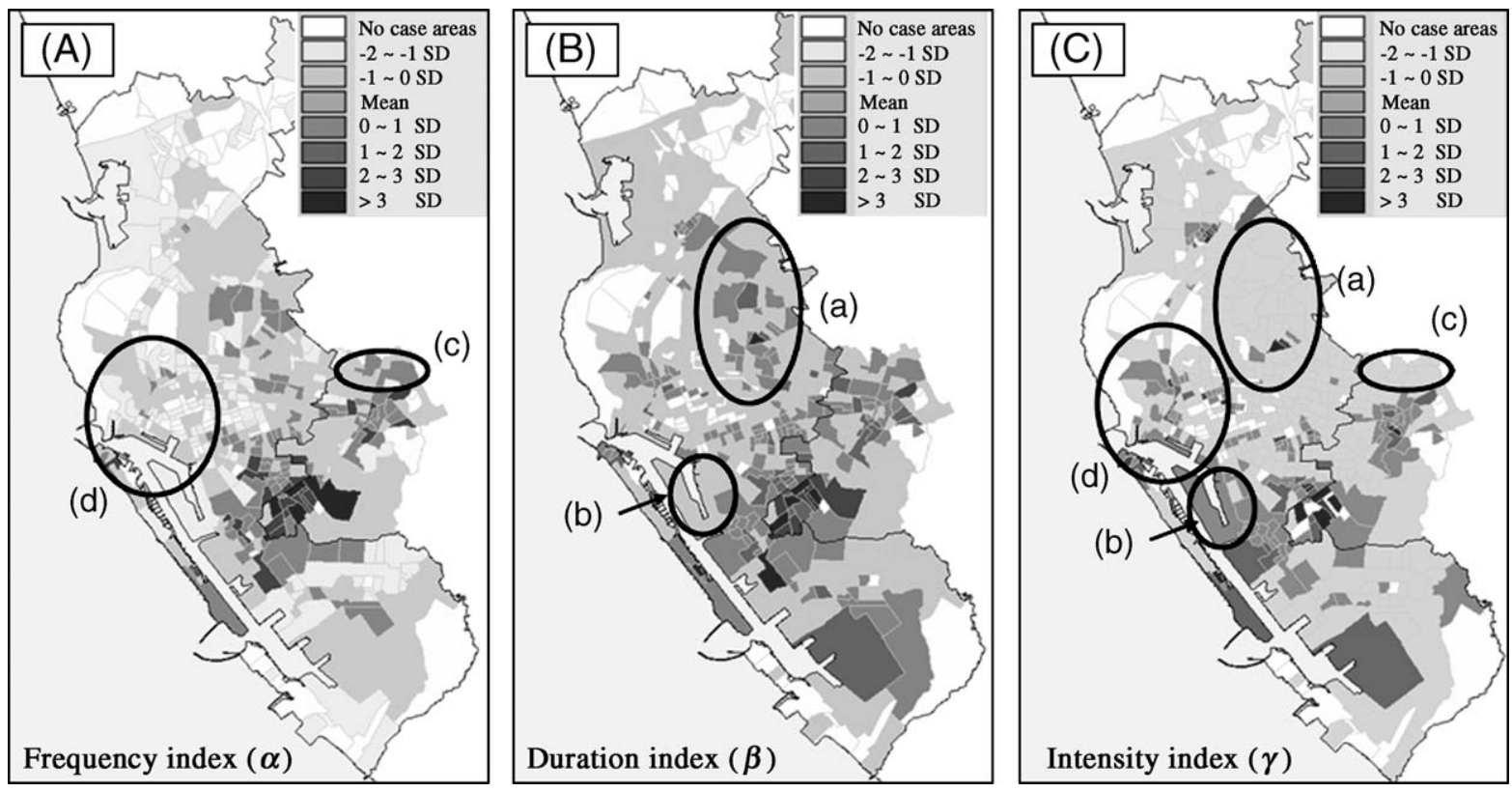

Fig. 4. Comparisons of spatial distribution of three temporal risk indices normalized by standard deviation.

classifications created from all possible combinations of the three temporal indices. The results are shown as in Table 4. Areas of type A are risk areas identified by LISA statistic as statistically significant high values of frequency, duration and intensity indices. Type B areas are those with only significant high frequency and duration indices and so on.

About $78.4 \%$ of $\operatorname{Li}(\mathrm{s})(425 / 542)$ are identified as type $\mathrm{H}$ with all three indices categorized as low, indicating that the DF cases has no significant temporal patterns in those areas. On the other hand, the other areas $(17.9 \%, 97 / 542)$ have different temporal risk patterns and possible meanings in public health domain are as follows.

- Type A areas: epidemic areas with highest severity, where might be the sources of spreading.

- Type B areas: the low intensity for this type may be resulted from mass spraying of insecticide, and insecticide-resistant mosquitoes might lead to high frequency and longer duration.

- Type C areas: blind spots due to unidentified source (s) or ineffective control measures might cause the high probability of case occurrences.

Table 4

Comparisons among different temporal risk types

\begin{tabular}{|c|c|c|c|c|c|c|}
\hline \multirow[t]{2}{*}{ Type } & \multicolumn{3}{|l|}{ Risk types } & \multirow[t]{2}{*}{ Count of $\mathrm{Li}$} & \multicolumn{2}{|l|}{ Population } \\
\hline & Frequency index, $\alpha$ & Duration index, $\beta$ & Intensity index, $\gamma$ & & $\begin{array}{l}\text { Density } \\
\left(100 \text { persons } / \mathrm{km}^{2}\right)\end{array}$ & Dengue cases \\
\hline A & High $0.255 \pm 0.08 *$ & High $4.608 \pm 3.32 *$ & High $3.179 \pm 2.01 *$ & 20 & 183.8 & 1054 \\
\hline B & High $0.241 \pm 0.08 *$ & High $2.919 \pm 1.56 *$ & $2.047 \pm 2.47$ & 11 & 102.37 & 332 \\
\hline $\mathrm{C}$ & High $0.183 \pm 006 *$ & $1.943 \pm 0.73$ & $1.173 \pm 1.21$ & 49 & 173.83 & 720 \\
\hline $\mathrm{D}$ & $0.106 \pm 006$ & High $2.085 \pm 0.83 *$ & High $2.191 \pm 1.28 *$ & 2 & 27.31 & 29 \\
\hline $\mathrm{E}$ & $0.110 \pm 0.05$ & $1.925 \pm 0.91$ & High $3.074 \pm 2.17 *$ & 10 & 269.71 & 94 \\
\hline $\mathrm{F}$ & High - & - & High - & 0 & 0 & 0 \\
\hline G & $0.100 \pm 0.04$ & High $2.134 \pm 0.79 *$ & $1.270 \pm 1.44$ & 5 & 237.03 & 47 \\
\hline $\mathrm{H}$ & $0.064 \pm 0.06$ & $1.133 \pm 0.83$ & $0.585 \pm 0.79$ & 425 & 85.7 & 2045 \\
\hline No cases & 0 & 0 & 0 & 20 & 183.8 & 0 \\
\hline Total & - & - & - & 542 & 93.4 & 4321 \\
\hline
\end{tabular}

1. The value of each temporal index is expressed as mean \pm standard deviation.

2. The symbol $(*)$ means that the value is statistically significant high $(p<0.05)$.

3. $\mathrm{Li}$ is the basic administration unit in Taiwan for urban planning and election. 
- Type D areas: the high intensity and long duration may be resulted from a severe epidemic, but low frequency likely results from these areas far from major epidemic foci.

- Type E areas: few but intense transmission pulses may have occurred, implying that the control measures might be effective.

- Type F areas: temporally sporadic but frequently interrupted transmission pulses, implying that the control measures might be effective briefly because of shorter duration but failed in the long run because of higher frequency of case-incidence. We did not find any area with this risk type, possibly because large-scale integrated measures had been implemented in this epidemic, combining mass spraying of insecticide and reduction of mosquito breeding had shorten the frequency and epidemic waves.

- Type G areas: this pattern can easily be ignored because of few cumulated cases. But the continuous
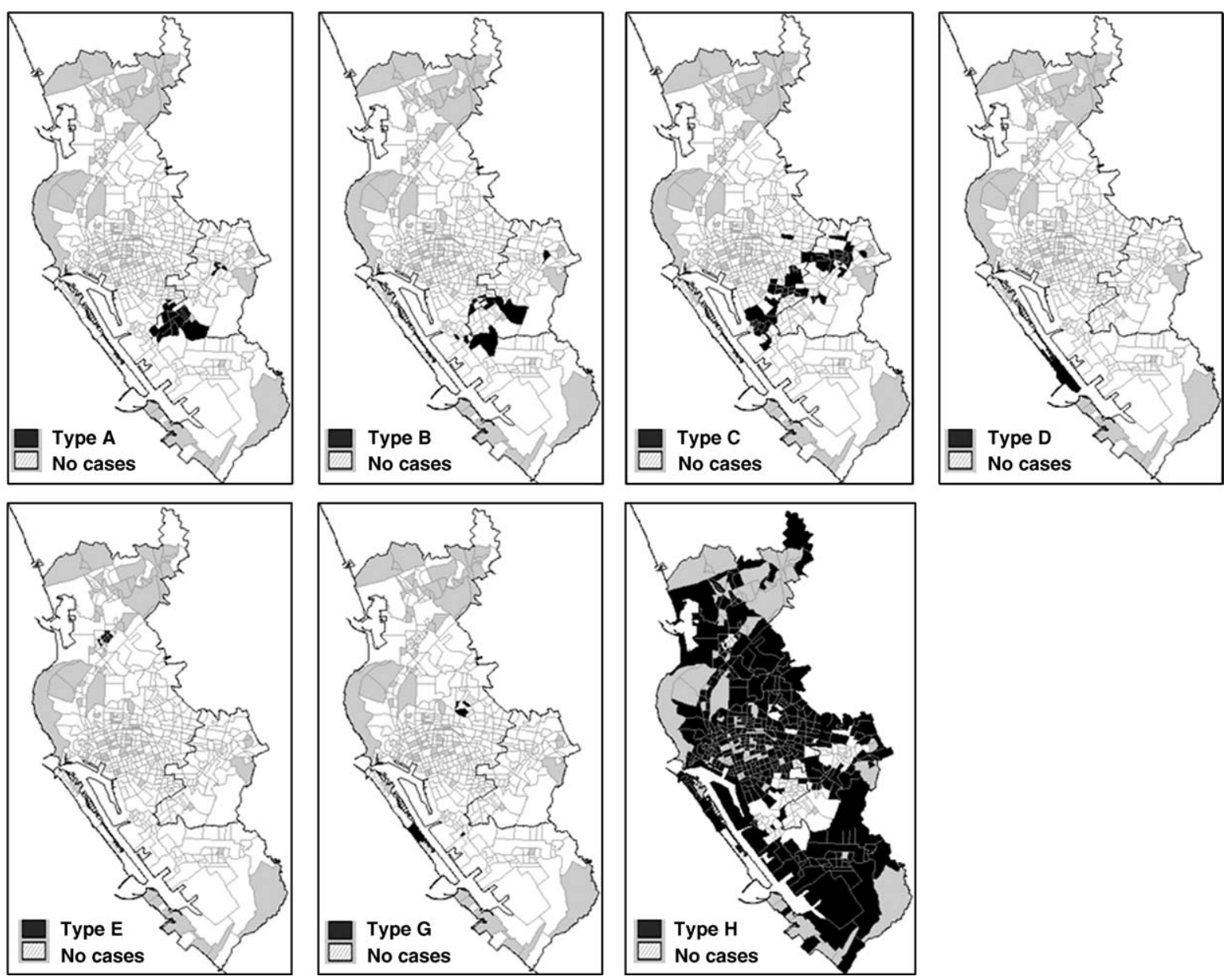

Fig. 5. Spatial risk areas with different temporal characteristics. presence of high duration might result in mild or silent transmission (Chen et al., 1996).

This novel approach in temporal-spatial modeling focused on the temporal risk characteristics opposed to the traditional use of case numbers. Fig. 5 shows spatial risk areas with different temporal characteristics. From observation, risk type A areas, surrounded by types B and $\mathrm{C}$ areas, had shorter duration or lower intensity implying that the risk type A areas may be the source of infection and where the epidemic had spread out (as shown in Fig. 6). Therefore, a transmission dynamics may be hypothesized, where the origin of this dengue epidemic most likely occurred in the most severe areas, risk type A, where delayed or ineffective control efforts may have allowed frequent, consecutive and intense occurrence of cases. Mass spraying of insecticide in risk type B areas may keep the transmission intensity relatively low even though cases occurred frequently 


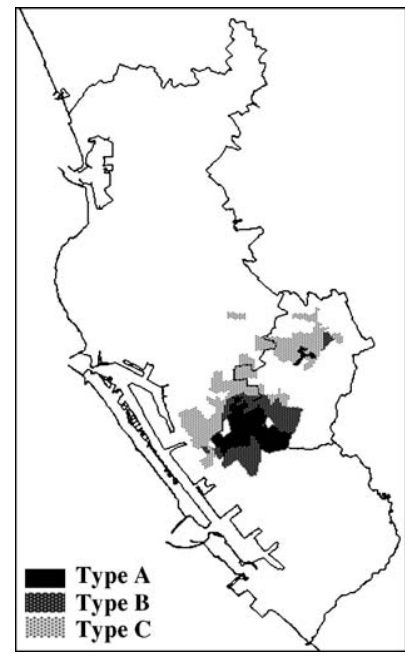

Fig. 6. Major epidemic foci in two-city areas.

and in succession due to insecticide-resistant mosquitoes. There are unidentified sources or blind spots of control measures in risk type $\mathrm{C}$ areas that cause higher case-occurrence probability.

Spatially, risk types D, E and G areas are located away from the major epidemic clusters (Fig. 5), giving support to virus movement by people as well as mosquitoes. The current belief is that movement of people facilitated the spread of dengue (Morrison et al., 1998) with transmission occurring locally (Edman et al., 1998; Harrington et al., 2005), which was observed in our case study. Further epidemiological investigation and entomological data may help in elucidating this trend. In conclusion, focusing on temporal factors allowed the identification of interactive spatial-temporal characteristics and provided a new perspective in explaining the severity.

\section{Discussion}

We have described a procedure for identifying spatial risk with different temporal characteristics of disease epidemics. The spatial risk maps with the three proposed temporal characteristics improve spatial clustering analysis, which focused mainly on case-incidence data obtained through passive surveillance. We are able to identify other case clusters when we factored in the temporal properties, such as case-incidence frequency and the number of cases that occurred within a certain time, or duration of epidemics. These factors give a comprehensive picture of the epidemic and therefore a more realistic representation of the risk.

The model relies on surveillance data because one major goal of this study is to enhance basic spatial modeling with the addition of temporal factors derived from an epidemic, while making the process simple and generally understood. A survey of public health practicians found that a widespread use of GIS was inhibited mostly by lack of understanding of the functions and training, even though they were well aware of what GIS can do (Cockings et al., 2004). This study uses basic surveillance data that is routinely collected in most countries, but with these three temporally defined risk characteristics, risk areas can be identified explicitly without using expensive, hightech equipments such as global positioning system (GPS) or remote sensing (RS). It will help in dispatching resources specifically to the most at-risk areas to prevent further cases from occurring or spreading.

However, the limitation of this study is its reliance on case-incidence data. In the study of arboviruses, data on disease vectors is important because human-mosquito contact is the main risk factor of dengue fever. Additional data on the principal mosquito vector $(A$. aegypti and Aedes albopictus), such as number of containers or control measures of breeding sites would give an estimate of vector presence. It would also be useful to determine the level of immunity circulating in the human population living in the study area, since many human dengue cases are asymptomatic. Human density, movement pattern and time would also give additional information in the analysis of dengue transmission patterns. Although the proposed model does not give answers to the questions of why the spatial patterns has occurred, it provides the health workers or epidemiologists with significant spatial risk levels taking account of temporal characteristics for generating hypothesis that can be investigated in further detail.

This application of the risk indices has demonstrated its effectiveness in identifying risk clusters with temporally defined characteristics and has improved the identification of case clusters. This approach can be applied to other infectious diseases, such as West Nile, to explore the temporal characteristics of the epidemic. Further applications of these risk indices will look into evaluating its effectiveness considering multi-year epidemics and at different locations.

\section{Concluding remarks}

Space and time are two important dimensions in describing epidemic dynamics and risk distribution. Methods based on either complicated statistical analysis or sophisticated surveillance systems are difficult to carry out in developing counties. Therefore, this paper attempted to use minimum data requirement and more 
straightforward statistical methods to capture major temporal characteristics of epidemic dynamic process, including frequency, duration and intensity. Different epidemic patterns can be clearly depicted through mapping these temporal risk indices. Rather than relying on mapping case number or incidence rate, this study enables the public health officers to comprehensively identify the risk areas. It also provides them with new and broader perspectives on temporal risk characteristics other than the traditional epidemic curves.

\section{Acknowledgements}

The authors sincerely thank the staff of Kaohsiung City and County Health Bureau and the Center for Disease Control in Taiwan (Taiwan-CDC) for their efforts in surveillance and vector control during the hard time of the dengue epidemic. The authors also thank anonymous referee's comments for clarifying the original manuscript. Neal Lin would like to express appreciation for the support from the Fulbright grant, sponsored by the Foundation for Scholarly Exchange in Taiwan.

\section{References}

Anselin L. Local indicators of spatial association-LISA. Geogr Anal 1995;27(2):93-115.

Bithell JF. An application of density estimation to geographical epidemiology. Stat Med 1990;9:691-701.

Bithell JF. Disease mapping using the relative risk function estimated from areal data. In: Lawson AB, Biggeri A, Bohning D, Lesaffre E, Viel J-F, Bertollini R, editors. Disease mapping and risk assessment for public health. New York: John Wiley \& Sons; 1999. p. $247-55$.

Bohra Alpana, Andrianasolo Haja. Application of GIS in modeling of dengue risk based on sociocultural data: case of Jalore, Rajasthan, India. Dengue Bull 2001;25:92-102.

Chen WJ, Chen SL, Chien LJ, Chen CC, King CC, Harn MR, et al. Silent transmission of the dengue virus in southern Taiwan. Am J Trop Med Hyg 1996;55(1):12-6.

Cockings S, Dunn CE, Bhopal RS, Walker DR. Users' perspectives on epidemiological, GIS and point pattern approaches to analyzing environment and health data. Health Place 2004;10(2):169-82.
Croner CM, Sperling J, Broom FR. Geographic information systems (GIS): new perspectives in understanding human health and environmental relationship. Stat Med 2000;15:1961-77.

Diggle PJ. Overview of statistical methods for disease mapping and its relationship to cluster detection. In: Elliott P, Wakefield J, Best N, Briggs D, editors. Spatial epidemiology: methods and applications. Oxford: Oxford University Press; 2000. p. 494.

DGBAS, Population and Housing Census Taiwan-Fukien Area, Republic of China, http://www.dgbas.gov.tw/, Nov. 2005 [in Chinese].

Duarte HH, Franca EB. Data quality of dengue epidemiological surveillance in Belo Horizonte, southeastern Brazil. Rev Saude Publica 2006;40(1):134-42.

Dunn CE, Kingham SP, Rowlingson B, Bhopal RS, Cockings S, Foy $\mathrm{CJ}$, et al. Analysing spatially referenced public health data: a comparison of three methodological approaches. Health Place 2001;7(1):1-12.

Earnest A, Chen MI, Ng D, Sin LY. Using autoregressive integrated moving average (ARIMA) models to predict and monitor the number of beds occupied during a SARS outbreak in a tertiary hospital in Singapore. BMC Health Serv Res 2005;11; 5(1):36.

Edman JD, Scott TW, Costero A, Morrison AC, Harrington LC, Clark GG. Aedes aegypti (Diptera: Culicidae) movement influenced by availability of oviposition sites. J Med Entomol 1998;35(4):578-83.

Giesecke J. Modern infectious disease epidemiology. London: Arnold; 2001. $280 \mathrm{pp}$.

Harrington LC, Scott TW, Lerdthusnee K, Coleman RC, Costero A, Clark GG, et al. Dispersal of the dengue vector Aedes aegypti within and between rural communities. Am J Trop Med Hyg 2005;72(2):209-20.

Maheswan Ravi, Haining Robert P. In: Ravi M, Massimo C, editors. Basic issues in geographic analysis, GIS in public health practice. CRC PRESS (Electric Media); 2004. 287 pp.

Morrison AC, Getis A, Santiago M, Rigau-Perez JG, Reiter P. Exploratory space-time analysis of reported dengue cases during an outbreak in Florida, Puerto Rico, 1991-1992. Am J Trop Med Hyg 1998;58(3):287-98.

Wilder-Smith A, Foo W, Earnest A, Sremulanathan S, Paton NI. Seroepidemiology of dengue in the adult population of Singapore. Trop Med Int Health 2004;9(2):305-8 [Feb].

Wu MH, Chao DY, Wang WK, Lin YL, King CC. The epidemiology of dengue fever/dengue hemorrhagic fever in Kaohsiung during 2001-2002. Proceeding of Taiwan Public Health Conference; 2003. p. 103-18 [in Chinese].

Zeger Scott L, Irizarry Rafael A, Peng Roger D. On time series analysis of public health and biomedical data, Johns Hopkins University, Dept. of Biostatistics, Working papers; 2004. 54 pp. 